\title{
Les représentations de la Bretagne dans l'iconographie postale française au XXe siècle
}

\section{Grégory Aupiais}

\section{OpenEdition}

Journals

Édition électronique

URL : http://journals.openedition.org/abpo/1132

DOI : $10.4000 /$ abpo. 1132

ISBN : 978-2-7535-1497-3

ISSN : 2108-6443

Éditeur

Presses universitaires de Rennes

\section{Édition imprimée}

Date de publication : 20 mars 2005

Pagination : 33-41

ISBN : 978-2-7535-0125-6

ISSN : 0399-0826

Référence électronique

Grégory Aupiais, «Les représentations de la Bretagne dans l'iconographie postale française au XXe siècle ", Annales de Bretagne et des Pays de l'Ouest [En ligne], 112-1 | 2005, mis en ligne le 20 mars 2007, consulté le 01 mai 2019. URL : http://journals.openedition.org/abpo/1132 ; DOI : 10.4000/ abpo. 1132 


\title{
Les représentations de la Bretagne dans l'iconographie postale française au Xx ${ }^{\mathrm{e}}$ siècle
}

\author{
Grégory AuPIAIS \\ Doctorant, université Paris I Panthéon-Sorbonne
}

Les premières émissions de timbres-poste ne sont intervenues en France qu'après le $1^{\mathrm{er}}$ janvier 1849 , sous la II ${ }^{\mathrm{e}}$ République et l'abandon d'une symbolique exclusivement monétaire fut simplement contemporain de la Première Guerre mondiale avec la commercialisation en août 1917 d'une série de huit figurines vendues avec une surtaxe au profit des orphelins de guerre. Ces différentes évocations des familles des soldats disparus, des tranchées, de la résistance française et de la victoire, à travers notamment le Lion de Belfort et la Marseillaise de Rude, allaient de paire avec une volonté de séduire un public beaucoup plus large, bien au-delà des seuls philatélistes. Une opération caritative similaire fut d'ailleurs organisée au bénéfice de la Croix rouge en 1918, puis de nouveau pour les orphelins de guerre en 1922. Ce spectre thématique initial, plutôt limité, s'est ensuite progressivement élargi après les Jeux olympiques de Paris en 1924, jusqu'à se figer en neuf registres principaux à la fin des années trente, parallèlement à l'annualisation des calendriers d'émission ${ }^{1}$.

Au sein de ce corpus iconographique d'un genre nouveau, les séries dites " touristiques " sont très vite devenues parmi les plus volumineuses, au moins du point de vue quantitatif. Une première expérience de six timbres-poste représentant respectivement le pont du Gard, le port de La Rochelle, le Mont-Saint-Michel, la cathédrale de Reims et l'Arc de triomphe a ainsi été émise entre le 15 mars 1929 et le 29 août 1931 à la demande du Commissariat général au tourisme. Une dimension territoriale particulièrement étroite, voire sélective, à laquelle la Bretagne n'accéda d'ailleurs que plus tardivement. Toutefois, ces évocations de sites et de paysages caractéristiques, de monuments historiques ou encore des arts

1. Liste des principaux thèmes abordés par l'iconographie postale française au $\mathrm{xx}^{\mathrm{e}}$ siècle (par ordre alphabétique) : " armée ", " arts et lettres ", " événement historique ", " expositions, congrès, conférences ", " série courante ", " sciences et techniques ", " séries touristiques ", "vie économique et sociale ", "vie politique ". 
et traditions populaires sont-elles réellement assimilables à des outils efficients de valorisation régionale?

\section{Un siècle d'imagerie postale}

En l'espace d'un siècle, 3260 timbres-poste ont été émis en France métropolitaine, dont 129 autour du thème générique de la Bretagne. Une analyse superficielle pourrait sans doute amener à considérer un tel ratio comme négligeable. Il ne l'est pourtant pas, compte tenu de l'extrême diversité usuelle de l'imagerie postale et du caractère massif de sa diffusion. En effet, ces 3,9\% représentent en fait plus d'un milliard de figurines qui, équitablement réparties sur l'ensemble du territoire national, ont touché toutes les strates de la société française sans aucune exception. La Bretagne effectue sa toute première apparition dans ce corpus iconographique le $1^{\mathrm{er}}$ mars 1931 à l'occasion de l'émission d'un timbre-poste vendu avec une surtaxe au profit de la Caisse d'Amortissement de la dette publique, un organisme crée en 1926 à l'initiative du Président du Conseil Raymond Poincaré pour assainir la situation financière de l'État (Figure 1) ${ }^{2}$. Elle intervient seulement quelques mois après l'Arc de triomphe, dernier volet de la commande du Commissariat général au tourisme et constitue d'ailleurs la première évocation régionale réelle, car détachée cette fois d'un contexte monumental. Il ne s'agit cependant que d'une discrète allusion par le biais d'une coiffe traditionnelle, partagée de plus avec l'Alsace mais aussi Arles et Boulogne-sur-Mer qui contribuèrent plutôt à en atténuer la dimension identitaire. La deuxième apparition de la Bretagne, le 11 décembre 1933, avec une figurine à l'effigie d'Aristide Briand est encore moins significative. En effet, même si cet homme politique est né à Nantes, le 26 mars 1862, son destin national voire international ne se prêtait pas véritablement à une tentative de récupération régionale. En fait, il faut attendre 1935 pour découvrir un premier paysage, le quatrième de l'histoire postale française après les tranchées, Alger et Le Puy-en-Velay (Figure 2). Cependant cette "Rivière bretonne " comme l'indiquait la légende présente sur les projets initiaux n'est pas identifiable ${ }^{3}$. Elle n'a même aucune existence matérielle, car née de l'imagination du peintre et graveur Jean-Émile Laboureur. Ce dernier s'est peut-être inspiré de l'estuaire de la Vilaine, proche de la commune de Pénestin dans laquelle il résidait ${ }^{4}$. Cependant, il n'est pas impossible non plus que sa composition soit un assemblage d'éléments disparates totalement artificiel. La seule figurine véritablement " bretonne " de la III ${ }^{\text {e }}$ épublique, une vue panoramique du port de Saint-Malo, n'a en réa-

2. Les neuf figures sont extraites de l'inventaire dressé par Bernard LE LANN sur son site Internet : [http://www.phil-ouest.com/].

3. BRun, Jean-François (dir.), Le Patrimoine du timbre-poste français, Charenton-le-Pont, Flohic Éditions, 1998, p. 213.

4. CHAUVEZ-ANSQUER, Jeanne, « Jean-Émile Laboureur au Croisic, contribution à l'étude de l'œuvre du peintre-graveur nantais (1877-1933) ", Visions contemporaines, n $^{\circ}$ 9, numéro spécial, 1996, p. 93. 
lité pas été émise avant le 16 mai 1938 (Figure 3). Par la suite, cependant, la Bretagne devint l'un des sujets récurrents de l'iconographie postale. Rares sont, en effet, les années où aucun timbre-poste ne lui est pas consacré, au moins d'une manière indirecte. Il y en a eu même jusqu'à sept en 1962, quand Jacques Marette est nommé ministre des PTT et un total de treize quand il démissionne le 6 avril 1967; une longévité exceptionnelle pour la Ve République, qu'il partagea d'ailleurs avec Louis Mexandeau, un autre ministre des PTT. Cet ancien journaliste à France-Soir puis à Combat, né à Paris le 21 septembre 1922, devenu homme politique et notamment sénateur UNR de la Seine après la Seconde Guerre mondiale n'est pas particulièrement lié à la Bretagne, du moins en apparence. Il s'agit peut-être d'un hommage au passé de résistant de ce fidèle du général de Gaulle, à moins que cette concentration unique ne soit simplement l'expression d'un attachement personnel voire familial. Les six figurines de l'année 1995 sont intervenues, en revanche, dans un contexte tout à fait différent. Elles sont rattachées à un hommage national rendu au naturaliste Jean-Jacques Audubon dont l'enfance se déroula dans la commune de Couëron, près de Nantes. Toutefois, ces deux exemples restent exceptionnels. La plupart du temps, en effet, ces émissions se limitèrent à un ou deux timbres-poste, rarement plus de trois. Elles sont intervenues dans leur grande majorité sous la Ve République. En revanche, la répartition par septennat ne fait pas apparaître de disparités notables, autres que celles inhérentes à la longueur du mandat; raison pour laquelle ceux du général de Gaulle et de François Mitterrand se détachent assez nettement.

Les cent vingt-neuf timbres-poste évoquant la Bretagne et les Bretons recensés au $\mathrm{XX}^{\mathrm{e}}$ siècle se répartissent entre neuf registres thématiques différents. Cette dispersion n'est cependant que très superficielle car les figurines " touristiques " en concentrent près de $28,6 \%$. Toutefois, les " sciences et techniques " et les " arts et lettres " en rassemblent quand même respectivement 27,9 et $19,3 \%$. Une partition déjà esquissée sous la IV ${ }^{\mathrm{e}}$ République et que le régime suivant a plutôt contribué à figer, en accentuant même les contrastes. Avant 1944, en effet, le nombre de vignettes est trop faible pour qu'une démarche statistique, tout du moins quantitative, soit opérante. Ainsi, dans la majorité des cas, ces évocations régionales demeurent très indirectes. Il peut même paraître inopportun de rattacher à un territoire ces émissions de timbres-poste à l'effigie de personnages célèbres. Ces derniers disposent déjà d'une identité propre et autonome qui les dispense d'un tel ancrage géographique. Néanmoins, c'est pourtant ainsi que localement de tels événements philatéliques sont vécus, tant ces célébrations semblent être des éléments constitutifs d'une "petite mémoire " généralement hypertrophiée par l'intérêt touristique qu'elle est susceptible de représenter. Cette relative hétérogénéité ne se retrouve pas cependant dans l'éventail chromatique utilisé. Une palette de dix couleurs au sein de laquelle les tonalités marines, c'est-à-dire le bleu, le vert et le noir sont très largement dominantes. 


\section{Chronique d'une ambiguiité iconographique ou l'exemple d'un régionalisme jacobin}

Imagerie postale et identité régionale peuvent a priori sembler difficilement conciliables. En effet, la fonction première d'un timbre-poste est d'abord de matérialiser le paiement d'une taxe à la puissance publique par l'expéditeur d'une lettre ou d'un colis. Les timbres-poste constituent ainsi, avec les pièces de monnaie et les billets de banque, l'un des éléments anodins d'une vaste architecture symbolique par laquelle l'État régalien manifeste sa présence, comme la permanence de son action, au sein de la population. Ce caractère centralisateur marqué n'empêche pas toutefois la coexistence de deux grands modes d'expression sur le plan graphique. Le premier, à la fois le plus ancien et le plus massivement diffusé, regroupe l'ensemble des figurines destinées à un usage courant. Bien qu'elles soient structurellement assimilables aux pièces de monnaie, les thématiques utilisées s'avèrent en revanche beaucoup plus elliptiques. La République ne s'est ainsi affirmée que très tardivement dans ce corpus iconographique spécifique. Le profil de Marianne n'apparaît, en effet, qu'après la Seconde Guerre mondiale succédant à d'autres évocations politiquement moins marquées comme, par exemple, Cérès, la déesse romaine des moissons, ou Iris, la messagère des dieux de l'Olympe. Le second rassemble les timbres-poste dits de " collection ", c'est-à-dire élaborés à l'attention d'un public plus ciblé, sans être restreint aux seuls philatélistes. L'hétérogénéité des thèmes abordés ne doit pas cependant faire illusion. Cette diversité n'est qu'apparente car ils sont tous dédiés à la célébration d'un " génie français ", que ce soit sur le plan littéraire, artistique, scientifique ou technologique. Une problématique dans laquelle se trouve également insérée la valorisation du territoire national, dont la dimension régionale a cependant été très largement minorée.

En effet, la Bretagne en tant que région n'a fait l'objet que d'un petit nombre de timbres-poste. En l'espace d'un siècle, seulement onze figurines sur cent vingt-neuf lui ont été directement consacrées. Les émissions dites "touristiques " avec plus de $45 \%$ sont très largement dominantes. Toutefois, elles se limitent pour l'essentiel à des évocations du folklore et des costumes traditionnels, dont le caractère identitaire n'est pas sans doute des plus fédérateurs. Une tendance générale qu'il était difficile de contrecarrer avec un paysage factice ou un blason. Ainsi, les timbres-poste peuvent difficilement être envisagés comme des instruments de valorisation du patrimoine régional, tant du point de vue historique, que culturel et encore moins politique. Indépendamment d'une notion d'impact aussi subjective qu'aléatoire, l'imagerie postale apparaît surtout comme étant l'une des manifestations, parmi d'autres, du centralisme jacobin. Il était donc plutôt logique, par conséquent, qu'elle ne serve pas de support à l'expression visuelle d'une forme de régionalisme, fut-elle policée.

Les quatre émissions les plus chargées de symboles sont intervenues dans des circonstances bien particulières sur le plan politique. Les deux 
premières en 1943 dans un contexte général d'exaltation de l'identité régionale, que le régime de Vichy cherchait à ériger en un nouvel instrument de gouvernement. Il tenta en effet de marginaliser les départements, perçus comme des créations républicaines, au profit de la commune et de la région. Héritiers respectifs des paroisses et des provinces d'Ancien Régime, ces découpages administratifs étaient jugés moins abstraits et surtout moins synonymes de démocratie représentative. Le recours systématique à l'héraldique, voire d'une manière plus secondaire au costume traditionnel, permettait d'en assurer la promotion et d'en manifester le profond enracinement dans l'opinion publique, sur lequel devait se fonder le renouveau de la Nation (Figure 4). Bien que s'inspirant très largement des thèses de Charles Mauras, dont le maréchal Pétain lui-même utilisait volontiers la dialectique, l'État français n'en adopta pas du tout le volet décentralisateur et s'orienta timidement vers une déconcentration hiérarchiquement moins sujette à conséquences. Les deux autres figurines ont été émises à la fin des années 1970 dans un contexte politique nettement plus apaisé. Néanmoins, elles apportèrent leur petite contribution à la vaste campagne de promotion institutionnelle qui précéda la loi de décentralisation du 2 mars 1982. Pas de rappel historique cette fois, mais deux créations artistiques originales censées illustrer une marche vers l'avenir tempérée par la vision bucolique d'un petit port breton anonyme (Figures 5 et 6 ). Ces figurines demeurent cependant exceptionnelles, pour ne pas dire anecdotiques. En effet, la grande majorité se sont plutôt faites l'écho de l'indivisibilité de la nation française; le folklore apparaissant, à bien des égards, comme l'ultime concession culturelle du centralisme politique. De telles orientations ont d'ailleurs été communes aux quelques commémorations d'événements historiques recensées. Le choix exclusif de la Seconde Guerre mondiale était, en effet, une manière de magnifier la communauté de destins unissant la Bretagne à la France dans la douleur ou la résistance à l'oppression.

\section{Des panoramas départementaux}

Ainsi, c'est donc plutôt le cadre départemental qui semble servir de référence institutionnelle à l'iconographie postale, car plus de 91 \% des cent vingt-neuf timbres-poste émis autour du thème générique de la Bretagne au xx siècle s'y rattachent, au moins indirectement. Leur répartition géographique est cependant loin d'être équitable. Les deux départements de la Loire-Atlantique et de l'Ille-et-Vilaine, avec respectivement trente-six figurines, en rassemblent en effet plus de 52 \% ; le Finistère, le Morbihan et les Côtes-d'Armor se contentant de pourcentages beaucoup plus modestes, qui s'échelonnent de 21 à $12 \%$.

De telles inégalités spatiales se doublent également de disparités thématiques particulièrement prononcées, si bien qu'il est difficile de dégager d'une telle hétérogénéité une esquisse de cohésion régionale. Ce corpus iconographique se scinde principalement en deux grandes catégories, dont les multiples déclinaisons rendent toutefois la définition malaisée. La pre- 
mière regroupe les deux départements du Finistère et du Morbihan dont le caractère touristique semble être prépondérant. Une majorité de timbresposte lui sont, en effet, consacrés et constituent de 52 à 69 \% du total des émissions. Ils sont également plutôt dispersés du point de vue géographique et ont construit progressivement un panorama plutôt avantageux de ces deux départements.

Ce véritable " office du tourisme postal " privilégie logiquement les paysages caractéristiques et les vues d'ensemble, au détriment des monuments historiques dont l'identité propre rejaillit rarement sur l'ensemble du département. Ainsi pour le Finistère, après la pointe du Raz en 1946, c'est tour à tour Quimper, à deux reprises, Brest, Morlaix, Saint-Pol-de-Léon, Concarneau, Landévennec, Douarnenez, les monts d'Arrée et Guimiliau qui furent mis en exergue. Quant au Morbihan, il ne se limite pas comme en 1965 à la seule évocation des alignements de Carnac. En effet, ce sont d'abord les illuminations des remparts de Vannes qui donnèrent lieu à l'émission d'un timbre-poste, avant de retrouver des sujets plus classiques comme La Trinité-sur-Mer, Port-Louis, Auray avec la ville et la basilique Sainte-Anne, Le Palais à Belle-Île, Malestroit et enfin Lorient en 1992. L'impact réel de telles campagnes de promotion du patrimoine naturel et architectural était cependant plutôt aléatoire, d'autant que son étalement chronologique sur plusieurs décennies ne pouvait éviter un phénomène de dilution. De plus, elles contribuaient plutôt à fixer ces départements dans une fonctionnalité déterminée, presque ludique, dont ils eurent par la suite beaucoup de mal à s'extraire.

Pour les autres, en revanche, l'accent a davantage été mis sur les sciences et techniques, en ne les limitant pas aux seules activités maritimes. Ainsi, le secteur des télécommunications semble faire partie intégrante de l'imagerie postale des Côtes-d'Armor, par ailleurs très minoritaire, puisqu'elle ne représente guère plus de $9 \%$ des timbres-poste émis. Mais, en dépit de cette faiblesse numérique, le centre de télécommunications spatiales de Pleumeur-Bodou bénéficie d'une couverture philatélique particulièrement soignée. Avec trois émissions de timbres-poste entre 1962 et 1965, la silhouette caractéristique du radôme est devenue, en effet, un élément incontournable du paysage postal de ce département (Figure 7). Cette tendance " scientiste " semble être partagée également, par la Loire-Atlantique et l'Ille-et-Vilaine, avec cependant quelques différences. En effet, si dans les deux cas les figurines relevant de cette thématique spécifique constituent avec 33 et 41,6\% une part importante des émissions, elles sont équilibrées par des pourcentages non négligeables dédiés aux arts et lettres et non, comme pour les Côtes-d'Armor, au tourisme. Cependant, même entre ces deux départements des nuances importantes peuvent être observées. En effet, pour la Loire-Atlantique, ces évocations semblent surtout s'être focalisées sur les infrastructures routières, voire ferroviaires, et la construction navale. Le " paysage technologique " de l'Ille-et-Vilaine est lui davantage tourné vers le passé maritime, à travers notamment quelques-uns de ses héros fondateurs comme Jacques Cartier ou le commandant Charcot. Plus 
rares sont les figurines résolument tournées vers la modernité, parfois même contestée d'ailleurs, à l'image de l'usine marémotrice de la Rance. De tels choix thématiques ont cependant généré de notables inégalités spatiales. En effet, Nantes et son agglomération concentrent près de $61 \%$ des émissions. En ajoutant la ville de Saint-Nazaire, souvent présentée comme son avant-port le pourcentage des deux tiers est largement dépassé. L'Illeet-Vilaine se distingue dans cette logique fonctionnelle et administrative : en effet, c'est la ville de Saint-Malo, point d'ancrage de la mémoire maritime, qui semble avoir monopolisé l'attention au détriment de Rennes.

Une répartition de cette nature peut également concerner un personnage célèbre, vécu localement comme une véritable figure emblématique. Ainsi pour la Loire-Atlantique, la personnalité de Jules Verne a complètement écrasé le paysage littéraire et artistique, au point de ravaler les autres évocations au rang de l'anecdote. Elle se manifeste à travers des portraits de l'écrivain, ou le plus souvent par des allusions directes à certaines de ses ouvres parmi les plus connues comme 20000 lieues sous les mers ou Cinq semaines en ballon (Figure 8). C'est également l'une d'elles : Le Phare $d u$ bout $d u$ monde qui été utilisée pour marquer symboliquement l'entrée dans le III ${ }^{\mathrm{e}}$ millénaire (Figure 9). Un monolithisme qui contraste avec la diversité recensée en Ille-et-Vilaine, ou même la figure pourtant centrale de François-René de Chateaubriand n'a jamais réussi à s'imposer.

Les timbres-poste sont, par excellence, des objets du banal et du geste ordinaire. Cette présence prégnante, souvent associée à un quotidien sans relief, a d'ailleurs très largement contribué à les rendre transparents, jusqu'à les effacer des mémoires collectives. Pourtant, ils s'avèrent être " un terreau particulièrement fertile pour le décrypteur d'images ${ }^{5}$ ".

Imperceptibles témoins de leur temps, ils sont en effet des vecteurs permanents d'un ensemble de représentations, comme autant de fragiles tessères d'une vaste mosaïque à la gloire du "génie français ". La valorisation du territoire constituait assurément l'un des volets les plus ambigus de cette exaltation nationaliste et c'est la raison pour laquelle sans doute elle n'est intervenue qu'à l'extrême fin des années vingt, avant de connaître un essor sans précédent sous la IVe, puis la Ve République. Une imagerie postale au sein de laquelle la Bretagne occupe désormais une place à part entière, après des débuts aussi timides que discrets.

Le $\mathrm{XxI}^{\mathrm{e}}$ siècle sera-t-il synonyme d'un profond renouvellement de ce corpus iconographique? Avec neuf nouveaux timbres-poste émis depuis janvier 2001, la permanence de la présence bretonne semble relever de l'évidence. Toutefois, sur le court terme, les déséquilibres géographiques apparaissent plus marqués encore. La Bretagne, en tant que telle, ne fait l'objet que de deux figurines, tout comme le Finistère, alors que parallèlement le département de Loire-Atlantique en suscite directement ou indirectement

5. GERVEREAU, Laurent, Voir comprendre analyser les images, Paris, La Découverte, coll. " Guides Repères », 1997, p. 157. 
près de $55 \%$. Ces dernières se partagent également entre seulement trois registres thématiques différents : les "sciences et techniques", les "séries touristiques " et la "vie économique et sociale". De plus les deux premières, presque traditionnelles, se trouvent désormais concurrencées par des allusions à la civilisation des loisirs englobant le sport, le cinéma ou la gastronomie. Une telle évolution peut surprendre. En effet, la place dévolue aux régions dans l'architecture institutionnelle européenne laissait supposer de nouvelles orientations plus constructives, voire plus identitaires. Toutefois, il est sans doute beaucoup trop tôt pour déceler, dans ce qui n'est peut-être qu'un épiphénomène, l'esquisse d'une tendance profonde.

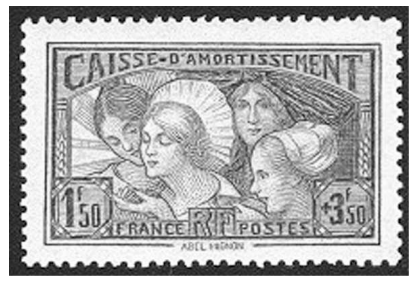

Figure 1 - $1931-Y T n^{\circ} 269$

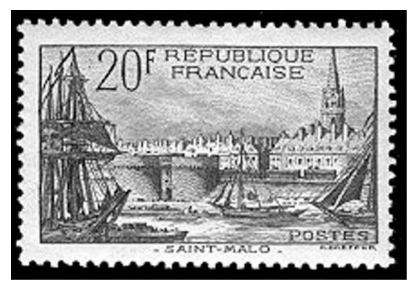

Figure 3 - $1938-Y T n^{\circ} 394$

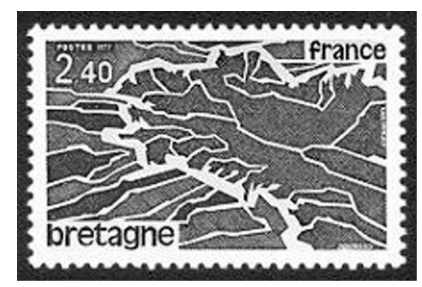

Figure 5 - $1977-Y T n^{\circ} 1917$

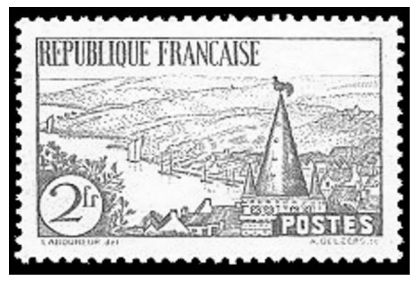

Figure 2 - $1935-Y T n^{\circ} 301$

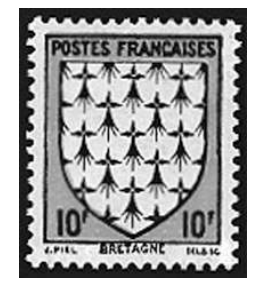

Figure 4 -

$1943-Y T n^{\circ} 573$

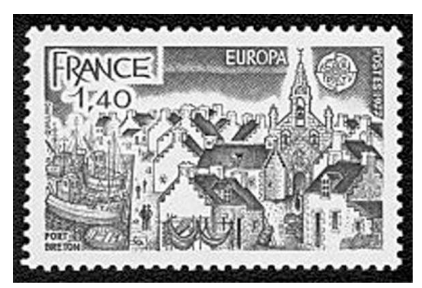

Figure 6 - $1977-Y T n^{\circ} 1929$ 


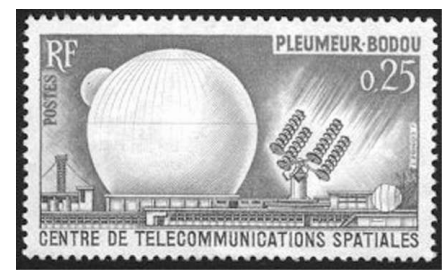

Figure $7-1962-Y T n^{\circ} 1360$

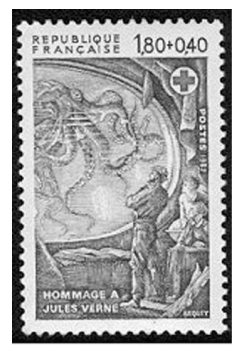

Figure 8 - 1982

$-Y T n^{\circ} 2248$

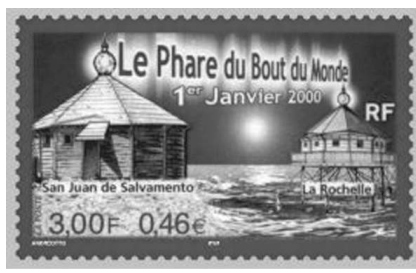

Figure $9-2000-Y T n^{\circ} 3294$

\section{RESUME}

La pérennité des émissions de timbres-poste, depuis leur création au milieu du $\mathrm{XIX}^{\mathrm{e}}$ siècle, peut surprendre. En effet, en dépit d'un processus de fabrication plutôt lourd, elles semblent survivre aux aléas de la conjoncture politique et économique. Une telle permanence aurait pu susciter, tout du moins éveiller, un intérêt historiographique; d'autant que ces fragiles symboles de la souveraineté nationale sont devenus de plus en plus complexes au fil des années. Dédiés à la célébration du " génie français ", ils possèdent également une dimension territoriale dont l'expression graphique n'est pas toujours dénuée d'ambiguïtés.

\section{ABSTRACT}

From their creation in the middle of the $19^{\text {th }}$ century to nowadays, the durability of the emissions of stamps may be surprise. In fact, in spite of a heavy makingprocess, they seem survive to the evolutions of the political and economic circumstances. These permanency could awaken an historiographical interest, all the more since these iconography convoyed by these fragile mediums of national sovereignty have become more and more complicated. Consecrated to a celebration of the "French genius", she have a territorial dimension too, whose graphic expression is not always devoid of ambiguities. 
